Groups Geom. Dyn. 5 (2011), 663-672

DOI $10.4171 / \mathrm{GGD} / 142$
Groups, Geometry, and Dynamics

(C) European Mathematical Society

\title{
Bernoulli actions and infinite entropy
}

\author{
David Kerr and Hanfeng Li
}

\begin{abstract}
We show that, for countable sofic groups, a Bernoulli action with infinite entropy base has infinite entropy with respect to every sofic approximation sequence. This builds on the work of Lewis Bowen in the case of finite entropy base and completes the computation of measure entropy for Bernoulli actions over countable sofic groups. One consequence is that such a Bernoulli action fails to have a generating countable partition with finite entropy if the base has infinite entropy, which in the amenable case is well known and in the case that the acting group contains the free group on two generators was established by Bowen.
\end{abstract}

Mathematics Subject Classification (2010). 37A35, 37A15.

Keywords. Entropy, Bernoulli action, sofic group.

\section{Introduction}

In [2] Lewis Bowen introduced a notion of entropy for measure-preserving actions of countable sofic groups admitting a generating partition with finite entropy. This measure entropy is defined relative to a given sofic approximation sequence for the group and thus yields a collection of numerical invariants in general. For a Bernoulli action with finite base entropy, Bowen showed that the sofic measure entropy is equal to the base entropy for every choice of sofic approximation sequence. As a consequence he was able to extend the entropy classification of Bernoulli actions in the amenable setting, due to Ornstein for $\mathbb{Z}$ and to Ornstein and Weiss more generally, to the class of all countable sofic groups having the property that any two Bernoulli actions with the same base entropy are conjugate, which includes all countable sofic groups containing an element of infinite order. Moreover, Bowen demonstrated that Bernoulli actions with nontrivial bases over a given countable sofic group containing the free group $F_{2}$ are all weakly isomorphic [1], which enabled him to conclude in [2] that Bernoulli actions with infinite entropy base over a countable sofic group containing $F_{2}$ do not admit a generating partition with finite entropy, thereby answering a question of Weiss.

In [4] the present authors extended Lewis Bowen's sofic measure entropy to general measure-preserving actions of a countable sofic group on a standard probability space by recasting the definition in operator-algebraic terms in a way that is reminis- 
cent of Rufus Bowen's approach to topological entropy for $\mathbb{Z}$-actions, which replaces the analysis of set intersections with the counting of $\varepsilon$-separated partial orbits. The main goal of [4] was to introduce a topological version of sofic measure entropy and establish a variational principle relating the two. In this note we respond to a question that Bowen posed to the authors by showing that, for countable sofic groups, a Bernoulli action with infinite entropy base has infinite entropy with respect to every sofic approximation sequence. The argument makes use of Bowen's finite entropy lower bound and is carried out by representing the dynamics in a topological way. It follows that the entropy of any Bernoulli action of a countable sofic group over a standard base is equal to the base entropy, independently of the sofic approximation sequence (Theorem 2.3). As a consequence, conjugate Bernoulli actions of a countable sofic group over standard bases have the same base entropy (Theorem 2.4), a fact that Bowen proved under the assumption that the bases have finite entropy or that the group is Ornstein, i.e., has the property that any two Bernoulli actions with the same base entropy are conjugate. Another consequence is that a Bernoulli action of a countable sofic group with an infinite entropy standard base does not admit a generating measurable partition with finite entropy (Theorem 2.6), which is well known for countable amenable groups and, as mentioned above, was established by Bowen for countable sofic groups containing $F_{2}$ using a different argument based on the weak isomorphism of Bernoulli actions. Note that there exist countable sofic groups which are not amenable and do not contain $F_{2}$. Indeed Ershov showed the existence of a countable nonamenable residually finite torsion group [3], Corollary 8.5 (see also [6]).

We now recall some terminology and notation pertaining to sofic measure entropy. We refer the reader to [4] for more details. Let $G$ be a countable sofic group. Let $\Sigma=\left\{\sigma_{i}: G \rightarrow \operatorname{Sym}\left(d_{i}\right)\right\}_{i=1}^{\infty}$ be a sofic approximation sequence for $G$, i.e., $\left\{d_{i}\right\}_{i=1}^{\infty}$ is a sequence of positive integers satisfying $\lim _{i \rightarrow \infty} d_{i}=\infty$ and the maps $\sigma_{i}$ into the permutation groups $\operatorname{Sym}\left(d_{i}\right)$ are asymptotically multiplicative and free in the sense that

$$
\lim _{i \rightarrow \infty} \frac{1}{d_{i}}\left|\left\{k \in\left\{1, \ldots, d_{i}\right\}: \sigma_{i, s t}(k)=\sigma_{i, s} \sigma_{i, t}(k)\right\}\right|=1
$$

for all $s, t \in G$ and

$$
\lim _{i \rightarrow \infty} \frac{1}{d_{i}}\left|\left\{k \in\left\{1, \ldots, d_{i}\right\}: \sigma_{i, s}(k) \neq \sigma_{i, t}(k)\right\}\right|=1
$$

for all distinct $s, t \in G$. The sofic measure entropy $h_{\Sigma, \mu}(X, G)$ of a measurepreserving action of $G$ on a standard probability space $(X, \mu)$ with respect to $\Sigma$ is defined, roughly speaking, by measuring the exponential growth as $i \rightarrow \infty$ of the maximal cardinality of $\varepsilon$-separated sets of approximately equivariant, approximately multiplicative, and approximately measure-preserving maps from $L^{\infty}(X, \mu)$ into $\mathbb{C}^{d_{i}}$, where the latter is equipped with the uniform probability measure. Instead of recalling the precise definition, which can be found as Definition 2.2 in [4], we will give here an equivalent formulation more suited to our purpose, which requires us to 
endow our measure-theoretic framework with topological structure in order to facilitate certain approximations. We thus suppose that $X$ is a compact metrizable space, $\mu$ is a Borel probability measure on $X$, and $\alpha$ is a continuous measure-preserving action of $G$ on $X$. The notation $\alpha$ will actually be reserved for the induced action on $C(X)$, so that $\alpha_{g}(f)$ for $f \in C(X)$ and $g \in G$ will mean the function $x \mapsto f\left(g^{-1} x\right)$, with concatenation being used for the action on $X$. Let $\mathcal{P}$ be a finite partition of unity in $C(X)$ and let $d \in \mathbb{N}$. On the set of unital homomorphisms from $C(X)$ to $\mathbb{C}^{d}$ we define the pseudometric

$$
\rho_{\mathcal{P}, \infty}(\varphi, \psi)=\max _{p \in \mathcal{P}}\|\varphi(p)-\psi(p)\|_{\infty} .
$$

Let $\sigma$ be a map from $G$ to the permutation group $\operatorname{Sym}(d)$ of the set $\{1, \ldots, d\}$. We also use $\sigma$ to denote the induced action on $\mathbb{C}^{d} \cong C(\{1, \ldots, d\})$, i.e., for $f \in \mathbb{C}^{d}$ and $s \in G$ we write $\sigma_{s}(f)$ to mean $f \circ \sigma_{s}^{-1}$. We write $\zeta$ for the uniform probability measure on $\{1, \ldots, d\}$, and $\|\cdot\|_{2}$ for the norm $f \mapsto \zeta\left(|f|^{2}\right)^{1 / 2}$ on $\mathbb{C}^{d}$. Let $F$ be a nonempty finite subset of $G, m \in \mathbb{N}$ and $\delta>0$. Define $\operatorname{Hom}_{\mu}^{X}(\mathcal{P}, F, m, \delta, \sigma)$ to be the set of all unital homomorphisms $\varphi: C(X) \rightarrow \mathbb{C}^{d}$ such that

(i) $|\zeta \circ \varphi(f)-\mu(f)|<\delta$ for all $f \in \mathcal{P}_{F, m}$,

(ii) $\left\|\varphi \circ \alpha_{s}(f)-\sigma_{s} \circ \varphi(f)\right\|_{2}<\delta$ for all $f \in \mathcal{P}$ and $s \in F$,

where $\mathcal{P}_{F, m}$ denotes the set of all products of the form $\alpha_{s_{1}}\left(p_{1}\right) \ldots \alpha_{s_{j}}\left(p_{j}\right)$ where $1 \leq j \leq m, p_{1}, \ldots p_{j} \in \mathcal{P}$, and $s_{1}, \ldots, s_{j} \in F$. The measure-preserving version of Proposition 4.11 in [4] (which can be established using the same argument) and the discussion in Section 5 of [4] together show that if $\mathcal{P}$ is dynamically generating in the sense that there is no proper $G$-invariant unital $C^{*}$-subalgebra of $C(X)$ containing $\mathcal{P}$, then

$$
h_{\Sigma, \mu}(X, G)=\sup _{\varepsilon>0} \inf _{F} \inf _{m \in \mathbb{N}} \inf _{\delta>0} \limsup _{i \rightarrow \infty} \frac{1}{d_{i}} \log N_{\varepsilon}\left(\operatorname{Hom}_{\mu}^{X}\left(\mathcal{P}, F, m, \delta, \sigma_{i}\right), \rho_{\mathcal{P}, \infty}\right)
$$

where $N_{\varepsilon}\left(\cdot, \rho_{\mathcal{P}, \infty}\right)$ denotes the maximal cardinality of an $\varepsilon$-separated subset with respect to the pseudometric $\rho_{\mathcal{P}, \infty}$ and $F$ ranges over all nonempty finite subsets of $G$.

Acknowledgements. The first author was partially supported by NSF grant DMS0900938, and the second author partially supported by NSF grant DMS-0701414. We are grateful to Lewis Bowen for pointing out the references [3], [6].

\section{Results}

Let $G$ be a countable sofic group and $(X, \mu)$ a standard probability space. Taking the product Borel structure on $X^{G}$ and the product measure $\mu^{G}$, we obtain a standard probability space $\left(X^{G}, \mu^{G}\right)$ on which $G$ acts by the shifts $g \cdot\left(x_{h}\right)_{h \in G}=\left(x_{g^{-1}}\right)_{h \in G}$, and we refer to this as a Bernoulli action. 
The following lemma is a direct consequence of (and is easily seen to be equivalent to) the lower bound for the sofic entropy of a Bernoulli action with finite entropy base that is part of Theorem 8.1 in [2]. Recall that the entropy of a measurable partition $Q$ of a probability space $(X, \mu)$ is defined by

$$
H_{\mu}(\mathcal{Q})=-\sum_{Q \in \mathcal{Q}} \mu(Q) \log \mu(Q) .
$$

Lemma 2.1. Let $G$ be a countable sofic group. Let $\Sigma=\left\{\sigma_{i}: G \rightarrow \operatorname{Sym}\left(d_{i}\right)\right\}_{i=1}^{\infty}$ be a sofic approximation sequence for $G$. Let $(X, \mu)$ be a standard probability space. Let $\mathcal{R}$ be a finite measurable partition of $X$. Then for every nonempty finite set $F \subseteq G$ and $\delta>0$ one has

$$
\begin{aligned}
& \limsup _{i \rightarrow \infty} \frac{1}{d_{i}} \log \mid\left\{\beta \in \mathcal{R}^{\left\{1, \ldots, d_{i}\right\}}:\right. \\
& \left.\quad \quad \sum_{\varphi \in \mathcal{R}^{F}}\left|\prod_{g \in F} \mu(\varphi(g))-\zeta\left(\bigcap_{g \in F} \sigma_{i}(g) \beta^{-1}(\varphi(g))\right)\right| \leq \delta\right\} \mid \\
& \geq H_{\mu}(\mathcal{R}) .
\end{aligned}
$$

Lemma 2.2. Let $G$ be a countable sofic group. Let $\Sigma=\left\{\sigma_{i}: G \rightarrow \operatorname{Sym}\left(d_{i}\right)\right\}_{i=1}^{\infty}$ be a sofic approximation sequence for $G$. Let $(X, \mu)$ be a standard probability space. Let $\mathcal{Q}$ be a finite measurable partition of $X$. Then $h_{\Sigma, \mu^{G}}\left(X^{G}, G\right) \geq H_{\mu}(\mathcal{Q})$.

Proof. We can identify $X$ with a Borel subset of $[0,1]$ such that the closures of the atoms of $\mathcal{Q}$ are pairwise disjoint. Then $\mu$ extends to a Borel probability measure $v$ on $\bar{X}$ such that $\mu(A)=v(A)$ for every measurable subset $A$ of $X$. It follows that the Bernoulli actions $\left(G, X^{G}, \mu^{G}\right)$ and $\left(G, \bar{X}^{G}, v^{G}\right)$ are measurably isomorphic. Thus $h_{\Sigma, \mu^{G}}\left(X^{G}, G\right)=h_{\Sigma, \nu^{G}}\left(\bar{X}^{G}, G\right)$. Denote by $\bar{Q}$ the partition of $\bar{X}$ consisting of $\bar{Q}$ for $Q \in \mathcal{Q}$. Then $H_{\mu}(\mathcal{Q})=H_{\nu}(\overline{\mathcal{Q}})$. Thus we may replace $X$ and $\mathcal{Q}$ by $\bar{X}$ and $\bar{Q}$ respectively. Therefore we will assume that $X$ is a closed subset of $[0,1]$ and $\mathcal{Q}$ is a closed and open partition of $X$. Equipped with the product topology, $X^{G}$ is a compact metrizable space. The shift action of $G$ on $X^{G}$ is continuous, and $\mu^{G}$ is a Borel probability measure on $X^{G}$. We write $\alpha$ for this action as applied to $C\left(X^{G}\right)$, following the notational convention from the introduction.

Denote by $p$ the coordinate function on $X$, i.e., $p(x)=x$ for $x \in X$. Then $\mathcal{P}:=\{p, 1-p\}$ is a partition of unity in $C(X)$ generating $C(X)$ as a unital $C^{*}$ algebra. Denote by $e$ the identity element of $G$. Via the coordinate map $X^{G} \rightarrow X$ sending $\left(x_{g}\right)_{g \in G}$ to $x_{e}$, we will also think of $\mathcal{P}$ as a partition of unity in $C\left(X^{G}\right)$. Then $\mathcal{P}$ dynamically generates $C\left(X^{G}\right)$, and so according to the introduction we have

$$
\begin{aligned}
& h_{\Sigma, \mu} G\left(X^{G}, G\right) \\
& \quad=\sup _{\varepsilon>0} \inf _{F} \inf _{m \in \mathbb{N}} \inf _{\delta>0} \limsup _{i \rightarrow \infty} \frac{1}{d_{i}} \log N_{\varepsilon}\left(\operatorname{Hom}_{\mu^{G}}^{X^{G}}\left(\mathcal{P}, F, m, \delta, \sigma_{i}\right), \rho_{\mathcal{P}, \infty}\right),
\end{aligned}
$$


where $F$ ranges over all nonempty finite subsets of $G$ and the pseudometric $\rho_{\mathcal{P}, \infty}$ on the set of unital homomorphisms $C\left(X^{G}\right) \rightarrow \mathbb{C}^{d_{i}}$ is given by

$$
\rho_{\mathcal{P}, \infty}(\varphi, \psi)=\max _{f \in \mathcal{P}}\|\varphi(f)-\psi(f)\|_{\infty}
$$

Take an $\varepsilon>0$ which is smaller than the minimum over all distinct $Q, Q^{\prime} \in \mathcal{Q}$ of the quantities $\min _{x \in Q, y \in Q^{\prime}}|x-y|$. Then it suffices to show

$$
\inf _{F} \inf _{m \in \mathbb{N}} \inf _{\delta>0} \limsup _{i \rightarrow \infty} \frac{1}{d_{i}} \log N_{\varepsilon}\left(\operatorname{Hom}_{\mu^{G}}^{X^{G}}\left(\mathcal{P}, F, m, \delta, \sigma_{i}\right), \rho \mathcal{P}, \infty\right) \geq H_{\mu}(\mathcal{Q})-\kappa
$$

for every $\kappa>0$.

So let $\kappa>0$. Let $F$ be a nonempty finite subset of $G, m \in \mathbb{N}$, and $\delta>0$. Note that, for each $f \in \mathcal{P}_{F, m}$, the value of $x \in X^{G}$ under $f$ depends only on the coordinates of $x$ at $g$ for $g \in F$. Thus we can find an $\eta>0$ such that whenever the coordinates of two points $x, y \in X^{G}$ at $g$ are within $\eta$ of each other for each $g \in F$, one has $|f(x)-f(y)|<\delta / 3$ for all $f \in \mathcal{P}_{F, m}$.

Take a finite measurable partition $\mathcal{R}$ of $X$ finer than $\mathcal{Q}$ such that each atom of $\mathcal{R}$ has diameter less than $\eta$. For each $R \in \mathcal{R}$ take a point $x_{R}$ in $R$. Let $\sigma$ be a map from $G$ to $\operatorname{Sym}(d)$ for some $d \in \mathbb{N}$. For each $\beta \in \mathcal{R}^{\{1, \ldots, d\}}$ take a map $\Theta_{\beta}:\{1, \ldots, d\} \rightarrow X^{G}$ such that for each $a \in\{1, \ldots, d\}$ and $g \in F$, the coordinate of $\Theta_{\beta}(a)$ at $g$ is $x_{\beta\left(\sigma(g)^{-1} a\right)}$. Then we have a unital homomorphism $\Gamma(\beta): C\left(X^{G}\right) \rightarrow \mathbb{C}^{d}$ sending $f$ to $f \circ \Theta_{\beta}$. Denote by $Z$ the set of $a$ in $\{1, \ldots, d\}$ such that $\sigma(e)^{-1} \sigma(g)^{-1} a=\sigma(g)^{-1} a$ for all $g \in F$. For every $\beta \in \mathcal{R}^{\{1, \ldots, d\}}, f \in \mathcal{P}$ and $a \in Z$, we have

$$
\begin{aligned}
\left(\Gamma(\beta)\left(\alpha_{g}(f)\right)\right)(a) & =\alpha_{g}(f)\left(\Theta_{\beta}(a)\right)=f\left(g^{-1} \Theta_{\beta}(a)\right) \\
& =f\left(\left(\Theta_{\beta}(a)\right)_{g}\right)=f\left(x_{\beta\left(\sigma(g)^{-1} a\right)}\right),
\end{aligned}
$$

and

$$
\begin{aligned}
(\sigma(g) \Gamma(\beta)(f))(a) & =(\Gamma(\beta)(f))\left(\sigma(g)^{-1} a\right)=f\left(\Theta_{\beta}\left(\sigma(g)^{-1} a\right)\right) \\
& =f\left(\left(\Theta_{\beta}\left(\sigma(g)^{-1} a\right)\right)_{e}\right)=f\left(x_{\beta\left(\sigma(e)^{-1} \sigma(g)^{-1} a\right)}\right) \\
& =f\left(x_{\beta\left(\sigma(g)^{-1} a\right)}\right),
\end{aligned}
$$

and hence

$$
\left(\Gamma(\beta)\left(\alpha_{g}(f)\right)\right)(a)=(\sigma(g) \Gamma(\beta)(f))(a) .
$$

When $\sigma$ is a good enough sofic approximation of $G$, one has $1-|Z| / d<\delta^{2}$, and hence

$$
\left\|\Gamma(\beta)\left(\alpha_{g}(f)\right)-\sigma(g) \Gamma(\beta)(f)\right\|_{2}<\delta
$$

for all $\beta \in \mathcal{R}^{\{1, \ldots, d\}}$ and $f \in \mathcal{P}$.

For each $\varphi \in \mathcal{R}^{F}$, denote by $Y_{\varphi}$ the set of $x$ in $X^{G}$ whose coordinate at $g$ is in $\varphi(g)$ for every $g \in F$. Then $\left\{Y_{\varphi}: \varphi \in \mathcal{R}^{F}\right\}$ is a Borel partition of $X^{G}$. For each 
$\varphi \in \mathcal{R}^{F}$ pick a $y_{\varphi} \in Y_{\varphi}$ such that the coordinate of $y_{\varphi}$ at $g$ is $x_{\varphi(g)}$ for each $g \in F$. By our choice of $\eta$ and $\mathcal{R}$, we have

$$
\sup _{x \in Y_{\varphi}} \sup _{f \in \mathcal{P}_{F, m}}\left|f(x)-f\left(y_{\varphi}\right)\right| \leq \delta / 3
$$

for every $\varphi \in \mathcal{R}^{F}$. For every $\beta \in \mathcal{R}^{\{1, \ldots, d\}}$ and $a \in\{1, \ldots, d\}$, define $\psi_{\beta, a} \in \mathcal{R}^{F}$ by $\psi_{\beta, a}(g)=\beta\left(\sigma(g)^{-1} a\right)$. Note that the coordinates of $\Theta_{\beta}(a)$ and $y_{\psi_{\beta, a}}$ at $g$ are the same for each $g \in F$. For every $\beta \in \mathcal{R}^{\{1, \ldots, d\}}$ and $\varphi \in \mathcal{R}^{F}$, one has

$$
\begin{aligned}
\left\{a \in\{1, \ldots, d\}: \psi_{\beta, a}=\varphi\right\} & =\bigcap_{g \in F}\left\{a \in\{1, \ldots, d\}: \psi_{\beta, a}(g)=\varphi(g)\right\} \\
& =\bigcap_{g \in F}\left\{a \in\{1, \ldots, d\}: \beta\left(\sigma(g)^{-1} a\right)=\varphi(g)\right\} \\
& =\bigcap_{g \in F} \sigma(g) \beta^{-1}(\varphi(g)) .
\end{aligned}
$$

Thus for every $\beta \in \mathcal{R}^{\{1, \ldots, d\}}$ and $f \in \mathcal{P}_{F, m}$ one has

$$
\begin{aligned}
\zeta(\Gamma(\beta)(f)) & =\frac{1}{d} \sum_{a=1}^{d} \Gamma(\beta)(f)(a)=\frac{1}{d} \sum_{a=1}^{d} f\left(\Theta_{\beta}(a)\right)=\frac{1}{d} \sum_{a=1}^{d} f\left(y_{\psi_{\beta, a}}\right) \\
& =\sum_{\varphi \in \mathcal{R}^{F}} f\left(y_{\varphi}\right) \zeta\left(\left\{a \in\{1, \ldots, d\}: \psi_{\beta, a}=\varphi\right\}\right) \\
& =\sum_{\varphi \in \mathcal{R} F} f\left(y_{\varphi}\right) \zeta\left(\bigcap_{g \in F} \sigma(g) \beta^{-1}(\varphi(g))\right) .
\end{aligned}
$$

Let $\tau$ be a strictly positive number satisfying $\tau<\delta / 3$ to be further specified in a moment. Set

$$
W=\left\{\beta \in \mathcal{R}^{\{1, \ldots, d\}}: \sum_{\varphi \in \mathcal{R} F}\left|\prod_{g \in F} \mu(\varphi(g))-\zeta\left(\bigcap_{g \in F} \sigma(g) \beta^{-1}(\varphi(g))\right)\right| \leq \tau\right\} .
$$

For every $\beta \in W$ and $f \in \mathcal{P}_{F, m}$ one has, since $0 \leq f \leq 1$,

$$
\begin{aligned}
\mu^{G}(f) & =\sum_{\varphi \in \mathcal{R}^{F}} \int_{Y_{\varphi}} f d \mu^{G} \approx_{\delta / 3} \sum_{\varphi \in \mathcal{R}^{F}} \mu^{G}\left(Y_{\varphi}\right) f\left(y_{\varphi}\right) \\
& =\sum_{\varphi \in \mathcal{R}^{F}} f\left(y_{\varphi}\right) \prod_{g \in F} \mu(\varphi(g)) \\
& \approx \delta / 3 \sum_{\varphi \in \mathcal{R}^{F}} f\left(y_{\varphi}\right) \zeta\left(\bigcap_{g \in F} \sigma(g) \beta^{-1}(\varphi(g))\right)=\zeta(\Gamma(\beta)(f)) .
\end{aligned}
$$

Therefore, when $\sigma$ is a good enough sofic approximation of $G$, the homomorphism $\Gamma(\beta)$ belongs to $\operatorname{Hom}_{\mu}^{X^{G}}(\mathcal{P}, F, m, \delta)$ for every $\beta \in W$. 
Let $\beta \in W$. We estimate the number of $\gamma \in W$ satisfying $\rho_{\mathcal{P}, \infty}(\Gamma(\beta), \Gamma(\gamma))<\varepsilon$. Note that

$$
\begin{aligned}
\rho_{\mathcal{P}, \infty}(\Gamma(\beta), \Gamma(\gamma)) & =\max _{a \in\{1, \ldots, d\}}\left|p\left(\Theta_{\beta}(a)\right)-p\left(\Theta_{\gamma}(a)\right)\right| \\
& =\max _{a \in\{1, \ldots, d\}}\left|x_{\beta\left(\sigma(e)^{-1} a\right)}-x_{\gamma\left(\sigma(e)^{-1} a\right)}\right| \\
& =\max _{a \in\{1, \ldots, d\}}\left|x_{\beta(a)}-x_{\gamma(a)}\right| .
\end{aligned}
$$

Thus $\beta(a)$ and $\gamma(a)$ must be contained in the same atom of $\mathcal{Q}$ for each $a \in\{1, \ldots, d\}$.

For $Q \in \mathcal{Q}$ denote by $\mathcal{R}_{Q}$ the set of atoms of $\mathcal{R}$ contained in $Q$. Thinking of $\beta$ as a partition of $\{1, \ldots, d\}$ indexed by $\mathcal{R}$, we see that $\left\{\bigcap_{g \in F} \sigma(g) \beta^{-1}(\varphi(g)): \varphi \in \mathcal{R}^{F}\right\}$ is a partition of $\{1, \ldots, d\}$. Let $Q \in \mathcal{Q}, R \in \mathcal{R}$, and $g_{1} \in F$. Then

$$
\sigma\left(g_{1}\right) \beta^{-1}(R)=\bigcup_{\substack{\varphi \in \mathcal{R} F \\ \varphi\left(g_{1}\right)=R}} \bigcap_{g \in F} \sigma(g) \beta^{-1}(\varphi(g)),
$$

and hence

$$
\zeta\left(\beta^{-1}(R)\right)=\zeta\left(\sigma\left(g_{1}\right) \beta^{-1}(R)\right) \approx_{\tau} \sum_{\substack{\varphi \in \mathcal{R} F \\ \varphi\left(g_{1}\right)=R}} \prod_{g \in F} \mu(\varphi(g))=\mu(R),
$$

and

$$
\begin{aligned}
\zeta\left(\beta^{-1}\left(\mathcal{R}_{Q}\right)\right) & =\zeta\left(\bigcup_{R \in \mathcal{R}_{Q}} \sigma\left(g_{1}\right) \beta^{-1}(R)\right) \approx_{\tau} \sum_{\substack{\varphi \in \mathcal{R}^{F} \\
\varphi\left(g_{1}\right) \in \mathcal{R}_{Q}}} \prod_{g \in F} \mu(\varphi(g)) \\
& =\mu\left(\bigcup \mathcal{R}_{Q}\right)=\mu(Q) .
\end{aligned}
$$

Similarly, we have $\zeta\left(\gamma^{-1}(R)\right) \approx_{\tau} \mu(R)$ for every $R \in \mathcal{R}$. The conclusion in the last paragraph can be restated as saying that $\beta^{-1}\left(\mathcal{R}_{Q}\right)=\gamma^{-1}\left(\mathcal{R}_{Q}\right)$ for every $Q \in \mathcal{Q}$. Hence the number of possibilities for $\gamma$ is bounded above by $\prod_{Q \in \mathcal{Q}} M_{\beta, Q}$, where listing the atoms of $\mathcal{R}_{Q}$ as $R_{1}, \ldots, R_{n}$ we have

$$
\begin{aligned}
M_{\beta, Q} & :=\sum_{j_{1}, \ldots, j_{n}}\left(\begin{array}{c}
\left|\beta^{-1}\left(\mathcal{R}_{Q}\right)\right| \\
j_{1}
\end{array}\right)\left(\begin{array}{c}
\left|\beta^{-1}\left(\mathcal{R}_{Q}\right)\right|-j_{1} \\
j_{2}
\end{array}\right) \ldots\left(\begin{array}{c}
\left|\beta^{-1}\left(\mathcal{R}_{Q}\right)\right|-\sum_{k=1}^{n-1} j_{k} \\
j_{n}
\end{array}\right) \\
& =\sum_{j_{1}, \ldots, j_{n}} \frac{\left|\beta^{-1}\left(\mathcal{R}_{Q}\right)\right| !}{j_{1} ! j_{2} ! \ldots j_{n} !},
\end{aligned}
$$

where the sum ranges over all nonnegative integers $j_{1}, \ldots, j_{n}$ such that $\mid j_{k} / d-$ $\mu\left(R_{k}\right) \mid \leq \tau$ for all $1 \leq k \leq n$ and $\sum_{k=1}^{n} j_{k}=\left|\beta^{-1}\left(\mathcal{R}_{Q}\right)\right|$. Setting $\xi(t)=-t \log t$ for $0 \leq t \leq 1$, for such $j_{1}, \ldots, j_{n}$ we have, by Stirling's approximation,

$$
\frac{\left|\beta^{-1}\left(\mathcal{R}_{Q}\right)\right| !}{j_{1} ! j_{2} ! \ldots j_{n} !} \leq C d \exp \left(\left(\sum_{k=1}^{n} \xi\left(j_{k} / d\right)-\xi\left(\left|\beta^{-1}\left(\mathcal{R}_{Q}\right)\right| / d\right)\right) d\right)
$$


for some constant $C>0$ independent of $\left|\beta^{-1}\left(\mathcal{R}_{Q}\right)\right|$ and $j_{1}, \ldots, j_{n}$. Since the function $\xi$ is uniformly continuous, when $\tau$ is small enough one has

$$
\sum_{k=1}^{n} \xi\left(j_{k} / d\right)-\xi\left(\left|\beta^{-1}\left(\mathcal{R}_{Q}\right)\right| / d\right)<\sum_{R \in \mathcal{R}_{Q}} \xi(\mu(R))-\xi(\mu(Q))+\kappa /|\mathcal{Q}|
$$

for all $j_{1}, \ldots, j_{n}$ as above. Therefore

$$
M_{\beta, Q} \leq C d(2 \tau d)^{\left|\mathcal{R}_{Q}\right|} \exp \left(\left(\sum_{R \in \mathcal{R}_{Q}} \xi(\mu(R))-\xi(\mu(Q))+\kappa /|\mathcal{Q}|\right) d\right)
$$

for every $Q \in \mathcal{Q}$, and hence

$$
\prod_{Q \in \mathcal{Q}} M_{\beta, Q} \leq C^{|\mathcal{Q}|} d^{|\mathcal{Q}|}(2 \tau d)^{|\mathcal{R}|} \exp \left(\left(H_{\mu}(\mathcal{R})-H_{\mu}(\mathcal{Q})+\kappa\right) d\right) .
$$

Now we have

$$
\begin{aligned}
& N_{\mathcal{E}}\left(\operatorname{Hom}_{\mu^{G}}^{X^{G}}(\mathcal{P}, F, m, \delta, \sigma), \rho_{\mathcal{P}, \infty}\right) \\
& \quad \geq|W| / \max _{\beta \in W} \prod_{Q \in \mathcal{Q}} M_{\beta, Q} \\
& \quad \geq|W| C^{-|\mathcal{Q}|} d^{-|\mathcal{Q}|}(2 \tau d)^{-|\mathcal{R}|} \exp \left(\left(-H_{\mu}(\mathcal{R})+H_{\mu}(\mathcal{Q})-\kappa\right) d\right) .
\end{aligned}
$$

Using Lemma 2.1 we thus obtain

$$
\begin{aligned}
& \limsup _{i \rightarrow \infty} \frac{1}{d_{i}} \log N_{\varepsilon}\left(\operatorname{Hom}_{\mu}^{X^{G}}\left(\mathcal{P}, F, m, \delta, \sigma_{i}\right), \rho_{\mathcal{P}, \infty}\right) \\
& \geq-H_{\mu}(\mathcal{R})+H_{\mu}(\mathcal{Q})-\kappa+\limsup _{i \rightarrow \infty} \frac{1}{d_{i}} \log \mid\left\{\beta \in \mathcal{R}^{\left\{1, \ldots, d_{i}\right\}}:\right. \\
&\left.\quad \sum_{\varphi \in \mathcal{R} F}\left|\prod_{g \in F} \mu(\varphi(g))-\zeta\left(\bigcap_{g \in F} \sigma_{i}(g) \beta^{-1}(\varphi(g))\right)\right| \leq \tau\right\} \mid \\
& \geq H_{\mu}(\mathcal{Q})-\kappa,
\end{aligned}
$$

as desired.

For a standard probability space $(X, \mu)$, the entropy $H(\mu)$ is defined as the supremum of $H_{\mu}(\mathcal{Q})$ over all finite measurable partitions $\mathcal{Q}$ of $X$. In the case $H(\mu)<+\infty$, the following theorem is Theorem 8.1 of [2] in conjunction with Theorem 3.6 of [4]. The case $H(\mu)=+\infty$ is a consequence of Lemma 2.2. When $G$ is amenable we recover a standard computation of classical measure entropy, in view of [5].

Theorem 2.3. Let $G$ be a countable sofic group. Let $\Sigma$ be a sofic approximation sequence for $G$. Let $(X, \mu)$ be a standard probability space. Then $h_{\Sigma, \mu^{G}}\left(X^{G}, G\right)=$ $H(\mu)$. 
As a consequence of Theorem 2.3 we obtain the following result, which was proved by Bowen in the case that $H(\mu)+H(v)<+\infty$ or $G$ is a countable sofic Ornstein group [2], Theorem 1.1 and Corollary 1.2. We note that it is not known whether there are countably infinite groups that are not Ornstein.

Theorem 2.4. Let $G$ be a countable sofic group. Let $(X, \mu)$ and $(Y, v)$ be standard probability spaces. If $\left(G, X^{G}, \mu^{G}\right)$ and $\left(G, Y^{G}, \nu^{G}\right)$ are isomorphic, then $H(\mu)=$ $H(v)$.

The next lemma follows from Proposition 5.3 of [2] (taking $\beta$ there to be the trivial partition) and Theorem 3.6 of [4].

Lemma 2.5. Let $G$ be a countable sofic group. Let $\Sigma$ be a sofic approximation sequence for $G$. Let $G$ act on a standard probability space $(X, \mu)$ by measurepreserving transformations. Let $\mathcal{Q}$ be a generating countable measurable partition of $X$. Then $h_{\Sigma, \mu}(X, G) \leq H_{\mu}(\mathcal{Q})$.

The following theorem is a consequence of Theorem 2.3 and Lemma 2.5. In the case that $G$ is amenable it is a well-known consequence of classical entropy theory, and in the case that $G$ contains the free group $F_{2}$ it was proved by Bowen in [2], Theorem 1.4. As mentioned in the introduction, there exist countable sofic groups that lie outside of these two classes [3], [6].

Theorem 2.6. Let $G$ be a countable sofic group. Let $(X, \mu)$ be a standard probability space with $H(\mu)=+\infty$. Then there is no generating countable measurable partition $\mathcal{Q}$ of $X^{G}$ such that $H_{\mu G}(\mathcal{Q})<+\infty$.

\section{References}

[1] L. Bowen, Weak isomorphisms between Bernoulli shifts. Israel J. Math. 183 (2010), 93-102.

[2] L. Bowen, Measure conjugacy invariants for actions of countable sofic groups. J. Amer. Math. Soc. 23 (2010), 217-245. Zbl 1201.37005 MR 2552252

[3] M. Ershov, Golod-Shafarevich groups with property (T) and Kac-Moody groups. Duke Math. J. 145 (2008), 309-339. Zbl 1162.20018 MR 2449949

[4] D. Kerr and H. Li, Entropy and the variational principle for actions of sofic groups. Invent. Math., DOI 10.1007/s00222-011-0324-9. arXiv:1005.0399v3

[5] D. Kerr and H. Li, Soficity, amenability, and dynamical entropy. Amer. J. Math., to appear.

[6] D. Osin, Rank gradient and torsion groups. Bull. London Math. Soc. 43 (2011), 10-16. Zbl 05853304 MR 2765544 
Received June 17, 2010; revised July 24, 2010

D. Kerr, Department of Mathematics, Texas A\&M University, College Station, TX 778433368, U.S.A.

E-mail: kerr@math.tamu.edu

H. Li, Department of Mathematics, SUNY at Buffalo, Buffalo, NY 14260-2900, U.S.A.

E-mail: hfli@math.buffalo.edu 